\title{
El reto de la formación ante el desarrollo de la Sociedad del Conocimiento en Europa
}

Jaume Pagès, Gabriel Ferraté, Josep Ma Duart

Arbor CLXXII, 678 (Junio 2002), 435-447 pp.

\section{La Sociedad del Conocimiento: una sociedad en cambio}

Querida, vivimos en una época de cambio, dijo Adán mientras acompañaba a Eva fuera del Paraíso.

William Ralph Inge Teólogo británico (1860-1954)

El conocimiento se está transformando en un elemento fundamental para el desarrollo económico y social. Se habla ya de la Sociedad del Conocimiento como una evolución de la sociedad industrial. En este contexto, la generación y la transmisión de conocimientos adquieren una relevancia inusitada. Es razonable pensar que la importancia que estas funciones puedan adquirir supere con mucho la que se les ha asignado en otros estadios del desarrollo social. En consecuencia, las instituciones que tradicionalmente han asumido estas funciones pueden ver, con razón, una gran oportunidad en la Sociedad del conocimiento. Sin embargo, la importancia del conocimiento puede también representar un reto. Con la importancia, puede aumentar la exigencia y puede aparecer la competencia. No es evidente que algunas instituciones tradicionales sean capaces de adaptarse a un incremento sustancial de exigencia, y en este caso podrían ser simplemente sustituidas.

La información, en tanto que vehículo de transmisión de conocimiento, es la pieza clave que transforma y dinamiza la sociedad de hoy. «La red es el mensaje» ${ }^{1}$ afirma el profesor Manuel Castells cuando expone 
que lo que él llama «la Galaxia Internet» crea un nuevo mundo de comunicación, diferente al generado en la época de la difusión masiva de la prensa escrita en Occidente que MacLuhan denominó «Galaxia Gutenberg».

Europa vio nacer, crecer y desarrollar las universidades como instituciones especializadas en la generación y transmisión del conocimiento. Es sin duda la región del mundo con la tradición universitaria de mayor solera. La irrupción de la sociedad del conocimiento -y de las tecnologías de la información- representa un reto para todas las sociedades, pero este reto tiene características singulares en el caso europeo, precisamente por el peso de la tradición.

\section{El impacto de la Sociedad del Conocimiento en la formación de las personas}

La educación debe adaptarse constantemente a los cambios de la sociedad, sin dejar de transmitir las adquisiciones, los fundamentos y los frutos de la experiencia humana.

Informe Jacques Delors (1996)

(La educación encierra un tesoro)

Los cambios generados por la Sociedad del Conocimiento provocan en las personas una demanda de formación que de respuestas a las necesidades de un mundo en constante proceso de adaptación. Ya no sirven para satisfacer la formación de los ciudadanos de hoy las instituciones de antaño, basadas en la simple transmisión de conocimiento y en ancladas metodologías, que otorgaban al profesor el papel predominante del proceso educativo. Los que forman la Sociedad del Conocimiento deben exigir a las instituciones educativas flexibilidad y calidad en los sistemas de formación para poder ejercer como ciudadanos de una sociedad en la que el aprendizaje es la base para afrontar los constantes procesos de cambio.

Avanzamos hacia una forma de aprendizaje basado en la red. El aprendizaje en red va más allá de la simple reproducción de los modelos tradicionales de formación que usan la tecnología para hacer lo mismo de siempre pero con cierto barniz de modernidad. Para aprender en red necesitamos formar nuevas habilidades en las personas y capacitarlas para aprender, desaprender y reaprender; necesitamos profesores y profesionales expertos en contenidos, con capacidad para difundir y compartir el conocimiento; y, sobre todo necesitamos instituciones flexibles con capa- 
cidad de organizarse para dar respuestas rápidas a las múltiples necesidades formativas de las personas en una sociedad en cambio.

Necesitamos una nueva formación para un mundo en red. Para ello debemos comunicar, informar y formar superando las barreras del tiempo y del espacio en un contexto global y con accesibilidad total. Nuestro objetivo debe ser el de poder transmitir más conocimientos a más personas y ser a la vez conscientes de que los conocimientos adquiridos son susceptibles de quedar obsoletos en poco tiempo. De ahí la necesidad de aprender a desaprender y a reaprender. Para ello las universidades y las instituciones de formación deben organizarse de tal forma que permitan a las personas acceder a la formación en cualquier momento y desde cualquier lugar ofreciendo asimismo la posibilidad de aprender al ritmo de las necesidades formativas de cada cual, de forma personalizada.

\title{
3. El papel de las Tecnologías de la Información y de la Comunicación en la formación
}

\author{
Technology is the answer! \\ But, what was the question? \\ The question was: is this a question? \\ Isn't this an answer? \\ Citado por James C. Taylor \\ Universtiy of Southern Queensland, Australia
}

La respuesta a la necesidad de una formación personalizada se basa crecientemente en la tecnología, y pasa por el uso adecuado de su potencial. Las tecnologías de la información y de la comunicación nos brindan la oportunidad de repensar y reformular los objetivos docentes para extraer de ellas el máximo partido. Pero no debemos caer en el error de pensar que el objetivo es el canal por el que vehiculamos la información o el conocimiento, ni la herramienta que usamos o el medio en el que nos situamos; el objetivo es la función que da sentido a lo que pretendemos hacer, que es formar. La tecnología, en todo caso, debe ser el medio y la formación el objetivo, es decir la función que pretendemos desarrollar.

Las Tecnologías de la Información y de la Comunicación pueden usarse en los procesos de formación como herramientas complementarias para facilitar la asimilación de los conocimientos o como soporte para su transmisión y desarrollo. El uso complementario de las TIC en la formación debe de ser el reto de todas las instituciones educativas, en especial el de las universidades. La irrupción de las tecnologías en las aulas uni- 
versitarias debe ir acompañado del rediseño de los programas de formación para aprovechar al máximo su potencial. El error de visión de muchos docentes universitarios reside en desestimar el valor resultante de la complementariedad de los modelos formativos que usan la tecnología -como por ejemplo internet- con el del modelo tradicional de la presencialidad. Pero ese error se agrava si no se comprende que el resultado óptimo de la complementariedad de los dos modelos reside en el diseño de una programación docente acorde con los medios tecnológicos que usamos y los objetivos formativos que pretendemos alcanzar.

El uso de las TIC como soporte en los procesos educativos tiene por resultado la formación virtual. Los actuales sistemas de formación virtual muestran los beneficios de la superación de las barreras del tiempo y del espacio. La virtualidad genera entornos reales que se pueden considerar reales en tanto que los efectos, las percepciones y las emociones que provocan, son reales en las personas ${ }^{2}$. La formación virtual facilita la flexibilidad en los procesos de comunicación, y conlleva la interactividad, la inmediatez y la personalización. El valor de la virtualidad radica también en su capacidad de provocar el replanteo de lo ya existente a partir de la recreación de la realidad. Al igual que en el uso complementario de las TIC en la formación, su uso como soporte necesita también de un rediseño de los programas docentes que sitúen la tecnología como medio y la formación como objetivo.

Los dos modelos expuestos anteriormente responden a diferentes retos de la educación ante la Sociedad del Conocimiento. Nos encontramos, por una parte, ante el reto de educar para la Sociedad del Conocimiento y por otra el de educar mediante la Sociedad del Conocimiento. La educación para la Sociedad del Conocimiento es la formación necesaria para asimilar y adaptarse a un mundo en cambio. Se trata de educar para abrir la mente de las personas y de las organizaciones, para flexibilizar las certidumbres y para apartar los miedos infundados sobre el posible caos generado por la constante evolución del mundo actual. Educar para la Sociedad del Conocimiento es también educar sobre las Tecnologías de la Información y de la Comunicación, no contra ellas o sin ellas, de ahí que los modelos de formación no puedan ser completamente ajenos al uso de las tecnologías.

Educar mediante la Sociedad del Conocimiento consiste en educar a través de un mundo en red. El futuro está en construir -por medio del impulso de la Sociedad del Conocimiento- universidades que trabajen en red en la consecución de sus objetivos; en personas que aprendan mediante el potencial de la red de conocimiento y que desarrollen nuevas aptitudes y capacidades para relacionarse, compartir y colaborar; y el 
reto de la pedagogía debe ser encontrar y aplicar aquellas metodologías de aprendizaje que aprovechen todo el potencial del mundo en red y ayuden a la creación de conocimiento mediante la red.

\title{
4. Europa, un caso singular: algunos retos específicos
}

\author{
Siempre hay un sueño en toda iniciativa, \\ pero, si el sueño dura, un día se hace realidad
}

Jean Monnet (1888-1979)

La singularidad de Europa, en cuanto a su papel en la Sociedad del Conocimiento reside en una realidad aparentemente paradójica: su ciudadanía, que es una de las de mayor nivel educativo del mundo, sufre debilidades y retrasos importantes en el uso de las tecnologías de la información y de la comunicación. Los niveles de penetración de internet en los hogares europeos o de uso del comercio electrónico no son los que cabria esperar de una sociedad avanzada como la europea. De ahí que el Consejo Europeo reunido en Lisboa en marzo de 2000, consciente de los retos a los que se enfrenta como resultado de la mundialización y de los desafíos de la nueva economía basada en el conocimiento, decide dotarse de la iniciativa eEurope ${ }^{3}$ que, en palabras de Romano Prodi, presidente de la Comisión Europea, es «la guía para modernizar nuestra economía»

La iniciativa eEurope se marca un ambicioso objetivo estratégico: «convertir Europa en la economía basada en el conocimiento más competitiva y dinámica del mundo, capaz de crecer económicamente de manera sostenible con más y mejores empleos y con mayor cohesión social» Todo objetivo debe ser ambicioso y, a la vez, debe marcar la ruta hacia una finalidad de mejora que sea mesurable. En este sentido y en el ámbito de la formación, el plan de acción preparado para el Consejo Europeo de Feria de junio de 2000, establece, como segundo objetivo de la iniciativa $e$ Europe, «invertir en las personas y en la formación» ${ }^{4}$ de acuerdo con la comunicación de la Comisión de mayo del 2000 denominada «eLearning, concebir la educación del futuro» ${ }^{5}$ que pretende estimular la evolución de los sistemas de educación y de formación hacia la sociedad del conocimiento a través de la movilización de las comunidades educativas y de los agentes económicos y sociales.

Iniciativas como eEurope y Elearning ponen de manifiesto la voluntad de nuestros gobernantes de habilitar los mecanismos y cauces nece- 
sarios para que el conocimiento fluya en nuestra sociedad y la convierta en competitiva a la vez que sostenible. El reto, como siempre, está en hacer realidad los objetivos marcados. Y especialmente en nuestro caso en facilitar que la tradición europea en la formación de calidad sea el motor de cambio que facilite los procesos de adaptación y mejora de nuestro sistema educativo.

Aparte de las iniciativas expuestas debemos citar la más relevante en el ámbito de la formación superior: la Declaración de Bolonia de junio de 1999 de los ministros de educación y de los rectores de las universidades de Europa. Dicha declaración manifiesta que «la Europa del Conocimiento se considera, cada vez más, como un factor indisoluble del crecimiento social y humano, así como un complemento indispensable para consolidar y enriquecer la ciudadanía europea, siendo capaz de ofrecer a los ciudadanos las competencias necesarias para afrontar los retos del nuevo milenio».

La Declaración de Bolonia es, sin duda, un hito en el proceso de construcción de un espacio europeo de formación superior. Los que la firman establecen cuatro ámbitos clave de actuación:

- La adopción de un sistema de titulaciones, comprensible y comparable, capaz de crear oportunidades de trabajo y de ser competitivo internacionalmente

- La creación de un sistema de dos ciclos principales: un no graduado y un graduado dirigido al mercado laboral

- El establecimiento de un sistema de créditos común

- El fomento de la movilidad de estudiantes, de profesores, de investigadores y de equipos administrativos, para favorecer la cooperación y la creación de aspectos específicamente europeos en los estudios superiores.

Europa tiene muchos retos que debe convertir en oportunidades para avanzar en la creación de la Europa del Conocimiento. Seguidamente proponemos algunos que afectan especialmente a la formación superior.

\section{El proceso de construcción de un espacio europeo de formación superior}

Europa puede y debe enorgullecerse de sus instituciones educativas. Los ciudadanos europeos gozamos de un alto nivel cultural y científico fruto de la labor desarrollada por las escuelas y las universidades a lo largo de siglos de historia. Hoy, inmersos cada vez más en un proceso consciente y palpable de construcción europea, debemos asumir el reto de 
El reto de la formación ante el desarrollo...

avanzar hacia un espacio europeo de formación superior de acuerdo con las directrices marcadas en la Declaración de Bolonia.

La riqueza y la tradición de las instituciones universitarias europeas debe ser la palanca en la que apoyarse para poder conseguir el movimiento necesario para la adaptación a la nueva realidad de la Sociedad del Conocimiento. La creación del espacio europeo de estudios superiores no debe observarse únicamente como el mecanismo para conseguir un modelo normativo común (títulos, créditos, etc) de las universidades europeas. El espacio europeo de estudios superiores va más allá y se construye de forma simultánea al establecimiento de las normas de base para el entendimiento. Debemos ser capaces de articular un nuevo modelo de universidad y de cooperación universitaria que nos conduzca a liderar en el mundo la capacidad de ayudar a aprender a nuestros alumnos, estén donde estén, y a investigar para innovar ayudando a los ciudadanos y a las empresas a mejorar sus niveles de competitividad.

La Declaración de Bolonia ha puesto las bases. Ahora les toca a las universidades y a sus equipos profesionales avanzar en la consecución de los objetivos. Nuestra experiencia como rectores nos ha mostrado la importancia de considerar los retos como oportunidades para mejorar y avanzar en nuestras instituciones. La construcción de un espacio de estudios superiores común en Europa no puede verse de otra forma que no sea un reto, un reto necesario para satisfacer las necesidades de nuestra Europa del Conocimiento.

\section{La calidad de la formación en Europa}

Los europeos debemos exigir calidad a nuestras instituciones formativas. Desde la escuela hasta la universidad la preocupación por la calidad debe ser la base para la mejora constante. La calidad en la educación superior es el resultado de la adaptación constante de las instituciones universitarias con la intención de satisfacer las necesidades formativas de una sociedad en cambio.

Las universidades europeas debemos afrontar el reto de garantizar la calidad de nuestros procesos de formación y de sus resultados potenciando tres aspectos básicos:

- La generación de dinámicas para la confección de planes estratégicos institucionales capaces de dinamizar la universidad y adaptarla a las necesidades de la sociedad de información

- El establecimiento de indicadores claros y fiables de valoración de la calidad en las universidades, fruto del trabajo compartido entre 
universidades, diferentes instituciones supragubernamentales y agencias de calidad.

- El estímulo de la comunicación y el trabajo cooperativo entre los agentes que forman parte de la comunidad universitaria europea -incluidos los diferentes sectores empresariales-, con la finalidad de detectar sus necesidades y establecer planes conjuntos de actuación.

Se trata en definitiva de ser capaces de mirar más allá de nuestras aulas y de nuestros claustros y situarnos en un ámbito europeo, en una sociedad en cambio, que nos demanda la creación de la Europa del Conocimiento. Para ello debemos actuar con rigor en el establecimiento de planes y de indicadores de valoración. Sólo el rigor en la consecución de niveles altos de calidad nos puede permitir avanzar en la consolidación de un modelo universitario europeo de referencia mundial y atraer así a estudiantes e investigadores de cualquier parte del planeta para aprender, enseñar e investigar en nuestras universidades.

La movilidad de estudiantes y de profesores como creación de espacio de formación europeo

Las universidades europeas disponen en la actualidad de gran experiencia en la aceptación y reconocimiento de la movilidad de estudiantes y de profesores. El reto de hoy es convertir la experiencia de la movilidad no en algo circunstancial fruto de convenios bilaterales sino en la forma de trabajar habitual en el marco del espacio europeo de formación superior.

Los claustros de las universidades europeas y sus clases deben estar formados por profesores y alumnos de diferentes países europeos. No tiene sentido un espacio formativo de educación superior en Europa si no compartimos nuestro conocimiento, ya sea como profesores, como investigadores, como administradores o como estudiantes. Y la movilidad o la presencia en las universidades no debe entenderse tan sólo de forma presencial; debemos aprovechar, una vez más, el potencial de las TIC y usar al máximo cualquier forma que favorezca el intercambio o movilidad, aunque ésta se realice sin moverse del país de residencia habitual.

La movilidad en el espacio de formación europeo, sea presencial o sea virtual, debe todavía superar algunos escollos, fruto muchas veces de mentalidades poco innovadoras o de administraciones demasiado cerradas en sí mismas. Sobre todo debe vencer los obstáculos de la hiperregulación a la que los títulos académicos están sometidos en Europa. Así 
El reto de la formación ante el desarrollo...

mismo, elementos como el reconocimiento de las garantías de seguridad social para las personas que se desplazan, los derechos de residencia por el hecho de formar parte de la comunidad universitaria, o el reconocimiento de las calificaciones obtenidas, entre otros, son realidades que debemos superar para garantizar la movilidad efectiva.

Las universidades europeas debemos observar la movilidad no sólo en nuestro ámbito geográfico, sino que debemos trabajar para ser focos de atracción de estudiantes y de investigadores de otras partes del mundo. Tenemos el potencial investigador, tenemos empresas multinacionales líderes, tenemos universidades de prestigio, pero quizás nos falta flexibilidad para generar los cambios que necesitamos y ser atractivos para cualquier investigador, profesor o estudiante de cualquier parte del mundo.

\section{La investigación como motor de creación de conocimiento compartido}

La investigación exitosa es el resultado del trabajo en equipo. Existen en Europa ejemplos de reconocimiento mundial en investigación, pero el reto para nuestro continente sigue siendo el de ser polo de atracción de investigadores. La relación entre las universidades y las empresas debe ser el factor clave de éxito para conseguir y mantener el prestigio en investigación.

Algunas empresas europeas como las del sector de la aeronáutica están dando un claro ejemplo de desarrollo conjunto entre empresas de diferentes países de productos líderes en el mercado mundial. La universidad europea no puede quedar al margen de la I+D que se desarrolla en las empresas. Europa dispone de las más potentes empresas del sector de las comunicaciones móviles y de la televisión digital del mundo, pero la mayoría de las universidades europeas no se benefician directamente de ello. Algunas están entrando recientemente en estos campos pero aún de forma insuficiente. El distanciamiento mantenido durante años entre universidad y empresa no puede continuar.

Las empresas europeas inician paulatinamente el camino hacia las universidades corporativas que tanto éxito tienen en otras partes del planeta. Ciertamente las grandes empresas multinacionales del sector de la tecnología tienen un elevado potencial investigador, más elevádo que el de cualquier universidad debido a su gran nivel de especificidad y también a los recursos que para ello destinan. La relación entre universidad y empresa para el desarrollo de programas de investigación no sólo es necesaria sino que de ella depende el futuro de Europa en el campo de la in- 
vestigación internacional, así como el futuro de competitividad de sus empresas multinacionales.

La iniciativa europea eLearning, así como la Declaración de Bolonia, remarcan la importancia del fomento y del apoyo a la investigación en Europa. Más concretamente la iniciativa eEurope, propone la mejora de la infraestructura actual de internet así como la creación de campus virtuales en red entre diferentes universidades y países. También se potencia la creación del «espacio Europeo de la Investigación» Europa no puede potenciar la investigación si antes no soluciona sus problemas de infraestructura en internet. Pero una vez más necesitamos un cambio de mentalidad en las instituciones educativas que de como resultado políticas abiertas de intercambio de personas, de movilidad de investigadores y de capacidad de crear proyectos compartidos de creación de conocimiento. Y especialmente necesitamos aprender a tomar decisiones con eficacia venciendo los intereses partidistas, ya sea de colectivos o organizaciones concretas como de gobiernos o países; sólo siendo rápidos y eficaces en las decisiones podremos avanzar en la construcción de un modelo de investigación competitivo en el mundo.

\section{La formación a lo largo de la vida}

Al contrario de lo que podíamos pensar, el reto de la formación a lo largo de la vida es un reto más para las instituciones de formación que para las personas. Las universidades debemos avanzarnos en la detección de necesidades formativas para dar respuestas en forma de programas formativos a los nuevos campos profesionales que se abren en el futuro. La crisis provocada por la falta de profesionales en el ámbito de la informática en Europa es un ejemplo de la poca visión de las universidades. No podemos permitirnos errores de estas características en el futuro y debemos trabajar detectando con tiempo aquellos campos en los que será necesaria una formación para la adecuación a nuevas necesidades profesionales, a nuevos servicios.

Los programas de formación también deben adaptarse a la nueva realidad de la formación a lo largo de la vida. La formación inicial ha de garantizar las capacidades mínimas para el desarrollo de una tarea profesional en un determinado campo, pero las universidades deben diseñar ofertas de posgraduado concretas, específicas, acordes con las necesidades de las empresas y capaces de solucionar los déficits de formación existentes. Muchos de esos programas deberán diseñarse pensando en que deben ser impartidos a gran número de personas; de ahí que el uso de 
El reto de la formación ante el desarrollo...

tecnología en la formación pueda ser una buena forma de dar respuesta a esa realidad.

En la formación a lo largo de la vida debemos orientarnos no sólo al desarrollo del conocimiento en las personas, sino especialmente al desarrollo de las competencias. La formación centrada en las competencias se basa en el conocimiento explícito, en el fomento de la experiencia y en el desarrollo de las aptitudes a través de las redes de relación social. Las competencias es lo que necesitamos adquirir las personas a lo largo de la vida. El reto de las universidades europeas consiste en detectar las competencias profesionales a desarrollar y ofrecerlas, conjuntamente con las empresas, a las personas.

\section{La flexibilidad en la organización del sistema de formación europeo}

La declaración de Bolonia pone también de manifiesto la necesidad de fomentar la flexibilidad en la organización del sistema europeo de formación. A pesar de ello sabemos que la flexibilidad sólo la conseguimos con instituciones universitarias capaces de conjugar su autonomía y tradición universitaria con la adaptación a las nuevas realidades organizativas de la Sociedad del Conocimiento. La universidad en Europa debe explotar al máximo el potencial de creatividad organizativa que suscita el desarrollo de modelos de formación con uso de tecnología digital. No podemos educar para y mediante la sociedad del conocimiento con modelos organizativos del pasado.

Las universidades deben modificar viejos hábitos y prácticas que han dado buenos resultados en otros tiempos pero que son un freno para el avance en la actualidad. Los responsables del gobierno en las universidades y sus equipos han de conducir el proceso de cambio hacia una organización flexible, basada en el diálogo, en la interacción entre todos los miembros de la comunidad universitaria, en la adopción de nuevos modelos de aprendizaje y de docencia, y en políticas activas de difusión del conocimiento. Los cambios deben empezar en el interior de cada universidad para pasar posteriormente a establecer relaciones flexibles en red con otras universidades.

Un sistema europeo de formación flexible debe permitir la movilidad, garantizar la calidad de la formación y especialmente reconocer las titulaciones y las asignaturas cursadas en cualquier otra universidad europea, luchando contra la hiperregulación académica existente. Debe además ser un sistema competitivo que forme para el desarrollo de una profesión y trabaje las competencias; debe ser un sistema que capte es- 


\title{
Jaume Pagès, Gabriel Ferraté, Josep $M^{a}$ Duart
}

tudiantes y profesores de otros sistemas del mundo; y, especialmente, debe ser un sistema a la medida de las personas que aprenden. Para ello deberá centrarse en la metodología de aprendizaje y en el uso adecuado de la tecnología que permita cumplir sus objetivos.

\section{Enseñar y aprender en Europa: un cambio de paradigma}

\author{
No se puede reformar la institución si no se han reformado \\ previamente los espíritus, \\ y no se pueden reformar los espíritus si previamente \\ no se ha reformado a institución.
}

Edgar Morin

La frase de Edgar Morin con la que iniciamos este apartado representa una paradoja atribuible a cualquier proceso de reforma institucional. Ante la imposibilidad lógica de resolver la paradoja Morin plantea que, en el caso de las instituciones formativas como las universidades, para resolver la situación «es necesario que los educadores se autoeduquen, y se educan escuchando las evidentes necesidades del siglo, de las que son portadores también sus estudiantes» Tenemos las respuestas a las preguntas en el interior de la propia institución. Únicamente debemos ser capaces de escuchar, de atender las necesidades de quienes se sientan en nuestras aulas, de nuestros compañeros de claustro y de nuestros administradores desde el gobierno de la universidad.

Europa y sus instituciones también deben escuchar a sus ciudadanos para asumir los retos de la satisfacción de sus necesidades. Y las universidades deben aprender y escuchar para construir nuevos modelos de formación. Las universidades europeas tienen ante sí la posibilidad de avanzar en la implementación de modelos de formación superior capaces de dar las respuestas necesarias a la construcción de la Sociedad del Conocimiento. Las universidades europeas deberían asímismo ser pioneras en la implementación de modelos de formación en los que las tecnologías de la telefonía móvil, de la televisión digital y de los sistemas de banda ancha fueran usadas para la consecución de los objetivos de aprendizaje. Para ello las universidades europeas, así como los gobiernos de los diferentes países, han de unir esfuerzos en la formación de los profesores, en el desarrollo de las infraestructuras tecnológicas y en el control de los operadores de telecomunicaciones para que hagan realmente accesible estos sistemas a la población en general. Escuchando las evidentes necesidades seremos capaces de generar respuestas. 
Podemos y debemos avanzar en la construcción de un nuevo paradigma en educación que estimule el desarrollo de dos actitudes básicas en las personas: la del aprendizaje a lo largo de la vida y la del aprendizaje en red. En una sociedad basada en el intercambio de información y en la creación de conocimiento, los hombres y las mujeres que la formamos debemos estar capacitados para el desarrollo de actitudes autónomas de aprendizaje. En palabras del sociólogo Castells debemos formar personas «autoprogramables", capaces de liderar su formación, de programar sus aprendizajes en función de sus necesidades personales y de compartir su información y su conocimiento en la red.

\section{Notas}

1 Castells, Manuel (2001) La Galaxia Internet, Plaza\&Janes. Barcelona.

2 Echevarría, Javier (2000) Un mundo virtual. Plaza \& Janes. Barcelona.

${ }^{3}$ COM (2000) eEurope, una sociedad de la información para todos. Consejo Europeo extraordinario de Lisboa, 23 y 24 de marzo.

4 COM (2000) Plan de Acción de eEurope preparado por el Consjo y la Comisión Europea para el Consejo Europeo de Feria, 19-20 de junio de 2000.

5 COM (2000) eLearning, concebir la educación del futuro, Bruselas 25.02.2000.

\section{Bibliografía}

Castells, Manuel (2001): La Galaxia Internet, Plaza \& Janes. Barcelona.

COMISIÓN EuROPEA (1996): Éducation-formation-recherche - Les obstacles à la mobilité transnationale - Livre vert. COM(96) 462, octobre 1996.

COMISIÓN EUROPEA (2000): eLearning, concebir la educación del futuro. Bruselas 25.02.2000.

Comisión EuRopea (2000): Plan de Acción de eEurope preparado por el Consejo y la Comisión Europea para el Consejo Europeo de Feria, 19-20 de junio de 2000.

Comisión EUROPEA (2000): Rapport de la Commission au Conseil et au Parlement Européen: Penser l'éducation de demain, promouvoir l'innovation avec les nouvelles technologies.

Comisión Europea (2001): Communication de la Commission au Conseil et au Parlement Européen. Plan d'action eLearning. Penser l'éducation de demain

Com 172, 28/03/2001.

Comisión Europea (2001) Rapport de la Commission. Les objectifs concrets futurs des systèmes d'éducation

Com 59, 31/01/2001.

DELORS, JACQUES (1996). La educación encierra un tesoro. Santillana. Madrid.

EcheVARría, JAVIER (2000) Un mundo virtual. Plaza \& Janes. Barcelona.

MORIN, EDGAR. (1998) "Sobre la reforma de la universidad" en Porta, Jaume (coord.) La universidad en el cambio de siglo. Alianza. Madrid. 